\title{
Validation of novel Japanese indication criteria and biomarkers among living donor liver transplantation recipients with hepatocellular carcinoma - a single center retrospective study
}

\author{
Akihiko Ichida, Nobuhisa Akamatsu, Kiyoshi Hasegawa \\ Artificial Organ and Transplantation Surgery Division, Department of Surgery, University of Tokyo, Tokyo 113-8655, Japan. \\ Correspondence to: Dr. Nobuhisa Akamatsu, Artificial Organ and Transplantation Surgery Division, Department of Surgery, \\ Graduate school of Medicine, University of Tokyo, 7-3-1 Hongo, Bunkyo-ku, Tokyo 113-8655, Japan. \\ E-mail: nakamats-tky@umin.ac.jp

\begin{abstract}
How to cite this article: Ichida A, Akamatsu N, Hasegawa K. Validation of novel Japanese indication criteria and biomarkers among living donor liver transplantation recipients with hepatocellular carcinoma - a single center retrospective study. Hepatoma Res 2020;6:54. http://dx.doi.org/10.20517/2394-5079.2020.59
\end{abstract}

Received: 18 Jun 2020 First Decision: 28 Jul 2020 Revised: 7 Aug 2020 Accepted: 18 Aug 2020 Published: 21 Aug 2020

Academic Editor: Guang-Wen Cao Copy Editor: Cai-Hong Wang Production Editor: Jing Yu

\begin{abstract}
Aim: To validate a novel Japanese indication criteria for liver transplantation (LT) for hepatocellular carcinoma $(\mathrm{HCC}$ ), i.e., the 5-5-500 criteria (nodule size $\leq 5 \mathrm{~cm}$ in diameter, nodule number $\leq 5$, and alfa-fetoprotein (AFP) value $\leq 500 \mathrm{ng} / \mathrm{mL}$ ) and the Japanese double eligibility criteria (DEC) (patients meeting the Milan or the 5-5-500 criteria) in the University of Tokyo cohort. The usefulness of biomarkers in predicting the recurrence of HCC was also verified.
\end{abstract}

Methods: The overall survival and recurrence rates of patients meeting the Milan, 5-5-500, and the Japanese DEC were compared among 153 patients who underwent living donor LT (LDLT) between 1996 and 2019. A receiveroperating characteristics curve analysis was conducted to evaluate the usefulness of AFP, lens culinaris agglutininreactive fraction of AFP, des-gamma-carboxy prothrombin, neutrophil-lymphocyte ratio, and the plateletlymphocyte ratio to detect recurrence.

Results: The 5-year recurrence rate for all patients, those meeting the Japanese DEC, 5-5-500 criteria, and the Milan criteria was $10.9 \%, 9.2 \%, 7.4 \%$, and $7.6 \%$, respectively. Compared with the conventional Milan criteria, the 5-5-500 criteria and the Japanese DEC could increase the number of eligible LDLT candidates by $6.1 \%$ and $11.4 \%$. Among five biomarkers, the area under the curve value of AFP was the highest (0.852).

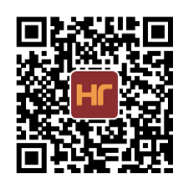


Conclusion: The results suggest that the 5-5-500 criteria and the Japanese DEC are the appropriate selection criteria for patients with HCC in LDLT. Among five biomarkers investigated, AFP was most reliable to predict HCC recurrence, which justified the utilization of AFP in the 5-5-500 criteria and the Japanese DEC.

Keywords: Indication criteria of liver transplantation for hepatocellular carcinoma, the 5-5-500 criteria, the Japanese double eligibility criteria, alfa-fetoprotein, the lens culinaris agglutinin-reactive fraction of alfafetoprotein, the des-gamma-carboxy prothrombin, the neutrophil-lymphocyte ratio, the platelet-lymphocyte ratio

\section{INTRODUCTION}

Expansion of the conventional Milan criteria ${ }^{[1]}$ has been debated over the last two decades ${ }^{[2-11]}$. Though recurrence of hepatocellular carcinoma (HCC) after liver transplantation (LT) has decreased since the proposal of the Milan criteria in 1996, it has been criticized as being too restrictive ${ }^{[11]}$. Initially, several extended criteria focused on expanding the upper limit of tumor size and number were reported ${ }^{[2-5]}$. In recent years, however, many authors have reported that an extended criteria should include the parameters reflecting the biologic behavior of the tumor ${ }^{[6-10]}$. With regard to the biomarkers incorporated into the expanded criteria, tumor markers such as alfa-fetoprotein (AFP) ${ }^{[7,10,12-15]}$ and des-gamma-carboxy prothrombin $(\mathrm{DCP})^{[6,8,15]}$ have been investigated by many researchers and recently, other markers such as the neutrophil-lymphocyte ratio (NLR ${ }^{[16,17]}$, the platelet-lymphocyte ratio (PLR $)^{[17]}$, and fluorine-18fluorodeoxyglucose positron emission tomography (FDG-PET) ${ }^{[18,19]}$ were reported to be useful for selection of the high risk group and prediction for recurrence. Another biomarker, the lens culinaris agglutininreactive fraction of AFP (AFP-L3), is widely used for the prediction of malignant biological behavior and poor prognosis of HCC patients in Japan, and may be a possible biomarker for recurrent HCC after $\mathrm{LT}^{[20-23]}$.

In Japan, living-donor liver transplantation (LDLT) has been the mainstay for end-stage liver disease patients with HCC due to the severe scarcity of deceased donors. While the gold standard has long been the Milan criteria, several center-oriented expanded criteria have been reported ${ }^{[3,6,8]}$. We proposed the Tokyo criteria in 2007, the detail of which was as follows; the number of tumors should be five or less, and the maximum diameter should be $5 \mathrm{~cm}$ or less, without distant metastasis nor vascular invasion ${ }^{[3]}$. Similarly, Kyoto and Kyushu advocated their own expanded criteria and included DCP as a biological marker ${ }^{[6,8]}$. These expanded criteria, however, had not been approved by the government, and those beyond the Milan criteria but still within each expanded criterion had to undergo LDLT as private practice, which led us to establish the government-approved expanded criterion. Most recently, the 5-5-500 criteria (nodule size $\leq$ $5 \mathrm{~cm}$ in diameter, nodule number $\leq 5$, and AFP value $\leq 500 \mathrm{ng} / \mathrm{mL}$ ) was established based on retrospective data analysis of the Japanese Liver Transplant Registry by our colleagues ${ }^{[24]}$. This expanded criteria was approved as the new national selection criteria for liver transplant candidates with HCC and started in August 2019. Now, the double eligibility criteria (DEC), Milan +5-5-500, has been adopted as the new indication criteria for Japanese patients with HCC.

The aim of the present study was to validate the Japanese DEC and the 5-5-500 criteria in our single-center cohort. In addition, the usefulness of biological markers (AFP, AFP-L3, DCP, NLR, and PLR) in predicting the recurrence of HCC after LT was also verified.

\section{METHODS}

\section{Patients}

From January 1996 until the end of 2019, a total of 563 adult patients underwent LDLT at the University of Tokyo Hospital. Among them, 153 patients were treated for HCC and were the subjects of the present study. Preoperative diagnosis of HCC was based on dynamic multi-detector computed tomography (MDCT) 
performed within a month before LT in all cases. Lesions presenting with typical radiological characteristics of classical HCC, that is, lesions with arterial phase enhancement and low density during the portal phase, were diagnosed as HCC to be counted and measured. In cases that underwent pretransplant locoregional treatments, only the size of the viable lesion was measured on the basis of MDCT before LDLT, and on the basis of pathological findings after LDLT. Essentially, we used the Milan criteria as a standard indication for LT for HCC; however, we allowed the expanded criteria, i.e., Tokyo criteria, in a private practice setting as mentioned above. Six cases exceeding the Tokyo criteria exceptionally, underwent LDLT in the early period. We did not use biomarkers such as AFP and DCP in patient selection.

\section{Donor selection and postoperative management}

Until 2015, the estimated graft volume to the recipient standard liver volume (SLV) ratio must be over $40 \%$ for LT at our institution. Since 2016, we have changed the threshold of the graft volume criteria to $35 \%$ of the recipient SLV. The left liver was the first choice for the graft if it satisfied the lower limit. Otherwise, right liver procurement was indicated if the estimated right liver graft volume was less than $70 \%$ of the donor's total liver volume, and a right lateral sector graft was used in selected cases. Details of donor evaluation and graft selection are described elsewhere ${ }^{[25]}$. The basic immunosuppression regimen comprised tacrolimus and steroid for all recipients, and the doses of each drug were gradually tapered over 6 months after LDLT. Our detailed postoperative recipient management including the immunosuppression protocol has been described elsewhere ${ }^{[26]}$. We do not modify immunosuppression for HCC recipients and do not use m-TOR inhibitors nor adjuvant chemotherapies. All patients were followed up at our department after LT according to the following protocol: monthly measurements of AFP and DCP, abdominal ultrasound performed every 3 months, and contrast-enhanced dynamic MDCT every 6 months. Recurrence was defined as the emergence of radiological findings in MDCT or magnetic resonance imaging compatible with typical HCC.

\section{Statistical analysis}

Categorical variables were expressed as number (\%) and continuous variables were expressed as median with range. NLR and PLR were calculated by dividing the number of neutrophils or platelets, respectively, by the number of lymphocytes. Patient overall survival and recurrence rates were calculated using KaplanMeier with Log rank test. A receiver-operating characteristics (ROC) curve analysis and Youden index were used to define the ideal cut-off values for AFP, AFP-L3, DCP, NLR, and PLR to detect recurrence. Univariate and multivariate analysis was performed using a Cox proportional hazards model to identify the predictors of recurrence. Factors with a $\mathrm{P}$ value less than 0.05 in a Cox proportional-hazard model as a univariate analysis were considered potential risk factors and further analyzed in a multivariate Cox model. The hazard ratio (HR) and 95\% confidence interval (CI) were calculated for each variable. Although seventeen variables listed in the table were examined as potential risk factors, AFP-L3 was excluded from multivariate analysis because of the quantity of missing data (AFP-L3 was not checked in 19 patients). Beyond the Milan, 5-5-500, and Japanese DEC were also excluded from multivariate analysis because they were not considered to be independent factors but composite factors having a strong relation to tumor number, size, and AFP value. All statistical calculations were performed using JMP Pro 15 (SAS Institute Inc., Cary, NC, USA). $P$ values less than 0.05 were considered statistically significant.

\section{RESULTS}

\section{Patient characteristics}

Recipient demographics are summarized in Table 1. There were 116 males and 37 females, with a median age of 56 (range, 37-67) years. The median model for end-stage liver disease score was 12 (range, 2-34). Hepatitis C virus positive cases comprised $57 \%$ of the cohort. Sixty percent $(92 / 153)$ had undergone locoregional treatments for HCC before LT, including 69 cases with transcatheter arterial chemoembolization, 31 with radiofrequency ablation, 23 with percutaneous ethanol injection therapy, and 14 with surgical 
Table 1. Characteristics of recipients with hepatocellular carcinoma

\begin{tabular}{|c|c|}
\hline & Recipients with hepatocellular carcinoma $(n=153)$ \\
\hline \multicolumn{2}{|l|}{ Pretransplant factors } \\
\hline Age (years) & $56(37-67)$ \\
\hline Gender (male/female) & $116 / 37$ \\
\hline Model for end-stage liver disease score & $12(2-34)$ \\
\hline Child-Pugh score & $9(5-14)$ \\
\hline \multicolumn{2}{|l|}{ Child-Pugh classification } \\
\hline Child A & $11(7 \%)$ \\
\hline Child B & $70(46 \%)$ \\
\hline Child C & $72(47 \%)$ \\
\hline \multicolumn{2}{|l|}{ Etiology } \\
\hline HBV & $44(29 \%)$ \\
\hline $\mathrm{HCV}$ & $85(56 \%)$ \\
\hline HBV, HCV, co-infection & $2(1 \%)$ \\
\hline Alcohol & $6(4 \%)$ \\
\hline Primary Biliary Cholangitis & $5(3 \%)$ \\
\hline Nonalcoholic Steatohepatitis & $4(3 \%)$ \\
\hline Other & $7(5 \%)$ \\
\hline \multicolumn{2}{|l|}{ Pretransplant treatments } \\
\hline Transcatheter arterial chemoembolization & $69(45 \%)$ \\
\hline Radiofrequency ablation & $31(20 \%)$ \\
\hline Percutaneous ethanol injection therapy & $23(15 \%)$ \\
\hline Liver resection & $14(9 \%)$ \\
\hline Proton therapy & $1(1 \%)$ \\
\hline \multicolumn{2}{|l|}{ Biomarker } \\
\hline $\operatorname{AFP}(\mathrm{ng} / \mathrm{mL})$ & $16(1-11999)$ \\
\hline AFP-L3 (\%) & 5 (undetectable-88) \\
\hline $\mathrm{DCP}(\mathrm{mAU} / \mathrm{mL})$ & $33(6-13248)$ \\
\hline NLR & $2.4(0.5-27.7)$ \\
\hline PLR & $74(18-578)$ \\
\hline Tumor number (pretransplant) & $1(0-14)$ \\
\hline Tumor size (pretransplant) $(\mathrm{cm})$ & $2(0-8)$ \\
\hline Tumor number (explants) & $2(1-19)$ \\
\hline Tumor size (explants) $(\mathrm{cm})$ & $2(0.5-11)$ \\
\hline \multicolumn{2}{|l|}{ Differentiation } \\
\hline Well differentiated & $67(44 \%)$ \\
\hline Moderately differentiated & $67(44 \%)$ \\
\hline Poorly differentiated & $8(5 \%)$ \\
\hline Necrosis & $11(7 \%)$ \\
\hline \multicolumn{2}{|l|}{ Intraoperative factors } \\
\hline Operation time (min) & $860(601-1890)$ \\
\hline Total blood loss $(\mathrm{mL})$ & $5390(568-53835)$ \\
\hline Graft volume $(\mathrm{g})$ & $554(300-880)$ \\
\hline Graft volume ratio to standard liver volume (\%) & $45(28-68)$ \\
\hline
\end{tabular}

HBV: hepatitis B virus; HCV: hepatitis C virus; AFP: alpha-fetoprotein; AFP-L3: lens culinaris agglutinin-reactive fraction of alphafetoprotein; DCP: des-gamma-carboxy prothrombin; NLR: neutrophil-to-lymphocyte ratio; PLR: platelet-to-lymphocyte ratio

resections. There were no cases of intentional downstaging or bridging therapy in this cohort. The median number and size of the tumor were 1 (range, $0-14$ ) and 2.0 (range, $0-8.0$ ) $\mathrm{cm}$ in the preoperative radiologic evaluation, while these were 2 (range, 1-19) and 2.0 (range, 0.5-11.0) $\mathrm{cm}$ in pathological examination of the explants. In histologic examination of explants, vascular invasion was confirmed in 21/153 (13.7\%) cases, and 67 (44\%), 67 (44\%), 8 (5\%), and 11 (7\%) patients had well-, moderately-, poorly-differentiated HCC, and necrosis, respectively. Two patients were diagnosed as having combined HCC-cholangiocarcinoma histologically. The median follow-up period after LDLT was 139 (range, 1-231) months for all patients. 


\section{Validation of the 5-5-500 criteria and the Japanese DEC}

The relationship of the Milan criteria, the 5-5-500 criteria, and the Japanese DEC is presented in the Venn diagram [Supplementary Figure 1]. The number of patients and patients with recurrence meeting each indication criteria was summarized in Supplementary Table 1. The recurrence rate was the lowest in patients meeting the 5-5-500 criteria (6.5\%) followed by the Milan criteria $(6.9 \%)$ and then the Japanese DEC (8.2\%). All criteria achieved the target of a recurrence rate below $10 \%$. When focusing on each area of the Venn diagram, the recurrence rate was the highest $(42.9 \%)$ in patients within the Milan but beyond the 5-5-500 criteria and in patients beyond the Japanese DEC. Meanwhile, the recurrence rate in patients within the 5-5-500 but beyond the Milan criteria was lower (20\%) than these patients. As for the comparison of the number of patients, the number of patients included in the 5-5-500 criteria was larger than that included in the conventional Milan criteria by eight ( $6.1 \%$ increase). In the Japanese DEC, 15 additional patients were included ( $11.5 \%$ increase). The overall survival and recurrence rate curves in patients meeting each indication criteria are presented in Figure 1. The 5-year overall survival and the 5-year recurrence rate of all the patients, patients meeting the Japanese DEC, 5-5-500 criteria, and Milan criteria was $76.9 \%, 77.9 \%, 79.0 \%$, and $76.2 \%$, and $10.9 \%, 9.2 \%, 7.4 \%$, and $7.6 \%$, respectively. There was no significant difference both in the 5-year recurrence and 5-year survival rates amongst each criterion.

\section{Usefulness of biomarkers in predicting the recurrence of $\mathrm{HCC}$}

The results of the ROC curve analysis for biomarkers is presented in Figure 2. Among the five biomarkers, the area under the curve (AUC) value of AFP was the highest (0.852). The sensitivity of AFP was also the highest (86.7\%). Meanwhile, the false-positive rate (1-specificity) of AFP-L3 was the lowest (8.3\%). Patient recurrence rate curves stratified by each biomarker using the cutoff value obtained from the ROC curve analysis are presented in Supplementary Figure 2. Though recurrence rate curves were well stratified with AFP, AFP-L3, and DCP $(P<0.0001)$, significant results were not obtained with NLR and PLR $(P=0.076$ and $=0.263$ respectively).

\section{Factors associated with HCC recurrence}

Risk factors associated with HCC recurrence were evaluated with univariate and multivariate analyses. Univariate analysis revealed that beyond the Milan, 5-5-500, and Japanese DEC were all significant predictors [Table 2]. Among these three criteria, the hazard ratio and $P$ value beyond the 5-5-500 criteria was the highest (7.99) and the smallest (0.0005), respectively. Except for factors associated with these three criteria, the high AFP value $\geq 60 \mathrm{ng} / \mathrm{mL}$, high AFP-L3 value ( $\geq 35 \%)$, high DCP value $(\geq 130 \mathrm{mAU} / \mathrm{mL})$, and large tumor size $(\geq 2.0 \mathrm{~cm})$ were all identified as significant predictors by univariate analysis. Among the five biomarkers evaluated, the hazard ratio and $P$ value of a high AFP value was the highest (11.50) and the smallest $(<0.0001)$, respectively. Multivariate analysis revealed that high AFP and DCP values were the independent significant predictors.

\section{DISCUSSION}

The results of the present study suggest that the 5-5-500 criteria and the Japanese DEC are appropriate and acceptable since the 5 -year recurrence rate in patients meeting these criteria were both below $10 \%$ in our cohort. Compared with the conventional Milan criteria, the 5-5-500 criteria and the Japanese DEC could increase the number of eligible LDLT candidates by $6.1 \%$ and $11.4 \%$, respectively. As for the usefulness of biomarkers in predicting the recurrence of HCC, AFP seems to be the most reliable. Though there were some missing data, AFP-L3 also seems promising.

In Japan, the national insurance system had restricted LDLT to those falling within the Milan criteria until recently, although some centers have been performing LDLT in private practice with a center-oriented expanded criteria that has achieved a 5-year patient survival over $80 \%$ and a 5 -year recurrence rate of $10 \%^{[27,28]}$. Consequently, a few patients had given up the chance for LDLT because of financial reasons, 

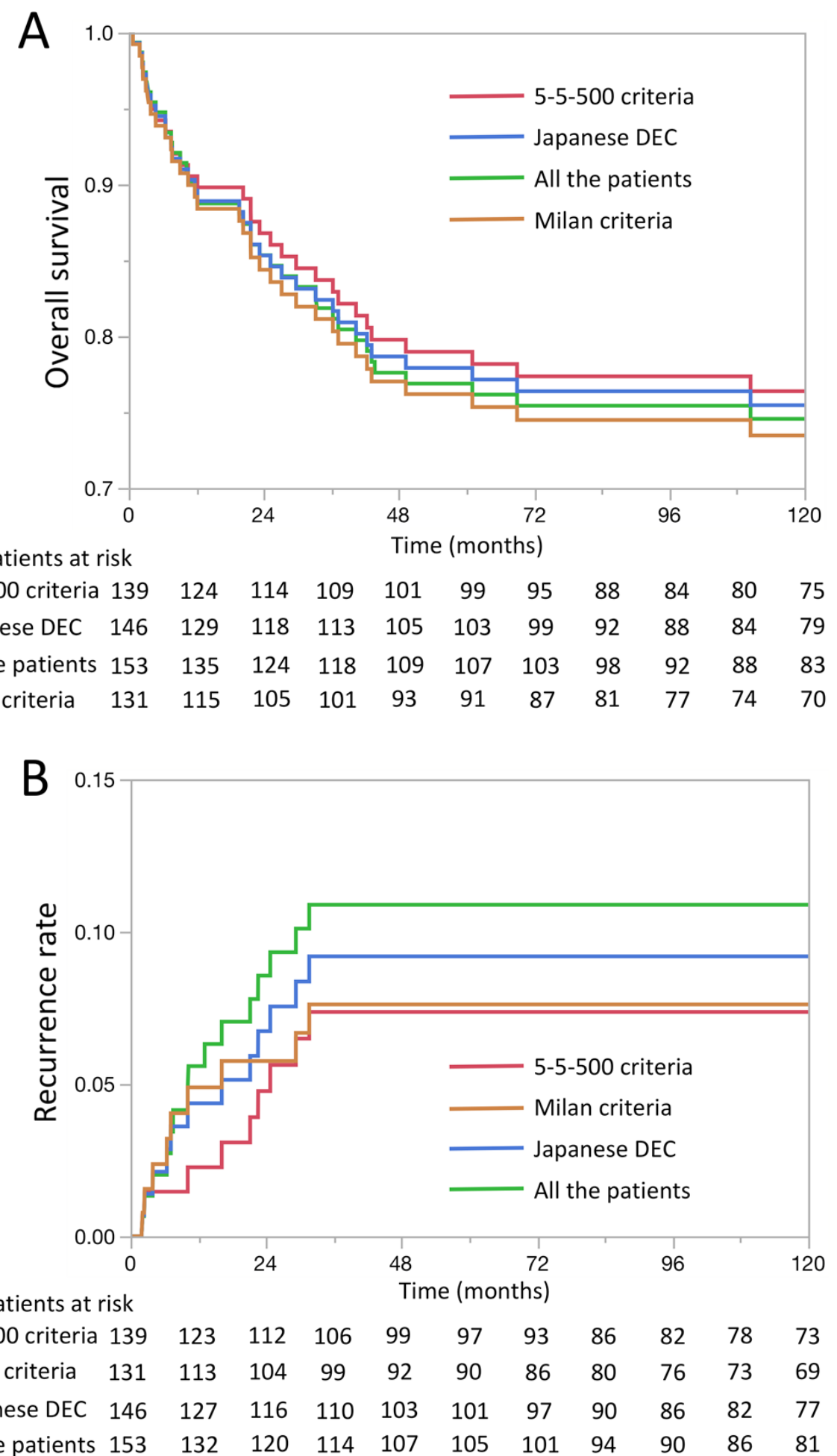

Figure 1. Overall survival $(A)$ and recurrence rate $(B)$ of patients meeting each indication criteria. DEC: double eligibility criteria

despite the potential of a live donor, and there has been strong demands to expand insurance coverage for those beyond the Milan criteria. When establishing government-approved expanded criteria, achieving a 5 -year recurrence rate of less than $10 \%$ and a 5 -year survival rate of over $70 \%$ seems reasonable and socially acceptable in the setting of LDLT for $\mathrm{HCC}^{[29]}$, which was achieved in the benchmark study by 


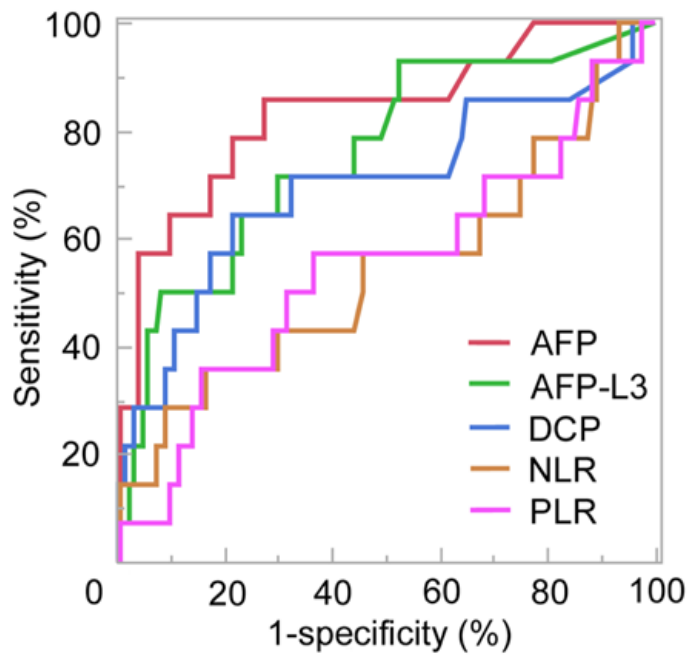

\begin{tabular}{lcccc}
\hline & Sensitivity (\%) & 1-specificity (\%) & AUC & Cutoff value \\
\hline AFP & 86.7 & 23.9 & 0.852 & $55 \mathrm{ng} / \mathrm{mL}$ \\
AFP-L3 & 50.0 & 8.3 & 0.754 & $35 \%$ \\
DCP & 66.7 & 20.3 & 0.719 & $132 \mathrm{mAU} / \mathrm{mL}$ \\
NLR & 40.0 & 15.9 & 0.564 & 5.4 \\
PLR & 33.3 & 15.2 & 0.533 & 138 \\
\hline
\end{tabular}

Figure 2. Receiver operating characteristic curves of true-positive (sensitivity) vs. false-positive (1 - specificity) rates with respect to recurrence plotted for AFP, AFP-L3, DCP, NLR, and PLR. AFP: alpha-fetoprotein; AFP-L3: lens culinaris agglutinin-reactive fraction of alpha-fetoprotein; DCP: des-gamma-carboxy prothrombin; NLR: neutrophil-to-lymphocyte ratio; PLR: platelet-to-lymphocyte ratio; AUC: area under the curve

Table 2. Univariate and multivariate analysis for recurrence

\begin{tabular}{|c|c|c|c|c|}
\hline \multirow{2}{*}{ Variables } & \multicolumn{2}{|c|}{ Univariate analysis } & \multicolumn{2}{|c|}{ Multivariate analysis } \\
\hline & HR (95\%Cl) & $P$ value & HR $(95 \% \mathrm{Cl})$ & $P$ value \\
\hline Age $\geq 60$ years & $1.02(0.28-2.99)$ & 0.970 & & \\
\hline Gender (male) & $1.16(0.37-5.09)$ & 0.816 & & \\
\hline MELD score $\geq 12$ & $1.22(0.44-3.64)$ & 0.703 & & \\
\hline Child-pugh score $\geq 9$ & $1.11(0.40-3.57)$ & 0.844 & & \\
\hline HBV infection & $2.00(0.70-5.58)$ & 0.187 & & \\
\hline HCV infection & $0.51(0.17-1.41)$ & 0.194 & & \\
\hline Pre-treatment therapies & $1.78(0.61-6.43)$ & 0.303 & & \\
\hline$A F P \geq 60 \mathrm{ng} / \mathrm{mL}$ & $11.50(3.64-50.51)$ & $<0.0001$ & $6.16(1.84-28.20)$ & 0.002 \\
\hline AFP-L3 $\geq 35 \% *$ & $8.72(2.97-25.57)$ & 0.0002 & & \\
\hline $\mathrm{DCP} \geq 130 \mathrm{mAU} / \mathrm{mL}$ & $7.22(2.56-23.26)$ & 0.0002 & $4.42(1.52-14.60)$ & 0.006 \\
\hline$N L R \geq 5$ & $2.47(0.83-6.85)$ & 0.101 & & \\
\hline PLR $\geq 140$ & $1.90(0.53-5.56)$ & 0.298 & & \\
\hline Tumor number $\geq 2$ (pretransplant) & $2.46(0.87-7.90)$ & 0.089 & & \\
\hline Tumor size $\geq 2.0 \mathrm{~cm}$ (pretransplant) & $9.06(1.82-164)$ & 0.004 & $4.74(0.90-87.59)$ & 0.071 \\
\hline Beyond Milan criteria* & $3.92(1.31-10.89)$ & 0.016 & & \\
\hline Beyond 5-5-500 criteria* & $7.99(2.67-22.22)$ & 0.0005 & & \\
\hline Beyond Japanese DEC* & $5.80(1.32-18.33)$ & 0.024 & & \\
\hline
\end{tabular}

${ }^{*}$ These variables were excluded from multivariate analysis. HR: hazard ratio; $\mathrm{Cl}$ : confidence interval; MELD score: model for end-stage liver disease score; HBV: hepatitis B virus; HCV: hepatitis C virus; AFP: alpha-fetoprotein; AFP-L3: lens culinaris agglutinin-reactive fraction of alpha-fetoprotein; DCP: des-gamma-carboxy prothrombin; NLR: neutrophil-to-lymphocyte ratio; PLR: platelet-to-lymphocyte ratio; DEC: double eligibility criteria 
Mazzaferro et al. ${ }^{[1]}$. In line with this recommendation, the 5-5-500 criteria was established with the intent to enable the maximal enrollment of candidates while securing a 5 -year recurrence rate below $10 \%$ and a 5 -year survival rate over $70 \%$ based on a retrospective data analysis of the Japanese nationwide survey ${ }^{[24]}$. Because the exclusion of patients within the Milan but beyond the 5-5-500 criteria seems not socially acceptable nor rationale, and considering the worldwide prevalence and acceptance of the Milan criteria, the Japanese DEC, Milan + 5-5-500, was adopted as the new indication criteria now in Japan.

In the present study, the 5-year recurrence and survival rate in patients meeting the 5-5-500 criteria and the Japanese DEC were superior to those socially accepted as mentioned above [Figure 1]. In addition, the number of LDLT candidates increased considerably using these criteria [Supplementary Figure 1 and Supplementary Table 1]. The outcomes of survival and recurrence were similar to our previous national report ${ }^{[24]}$ though the increase of LDLT candidates was a bit modest in the present study. Univariate analysis revealed that both beyond the 5-5-500 criteria and beyond the Japanese DEC were significant predictors of recurrence [Table 2]. Meanwhile, the recurrence rate was higher in patients beyond the Japanese DEC [Supplementary Figure 1 and Supplementary Table 1]. On the basis of these findings, we consider that the Japanese DEC are the appropriate selection criteria to maximize the number of LDLT candidates while securing acceptable outcomes. The major concern is that the recurrence rate was considerably high (42.9\%) in patients within the Milan but beyond the 5-5-500 criteria in the present study [Supplementary Table 1]. The exclusion of patients within the Milan criteria, however, seems not socially acceptable at present. In addition, when the Japanese DEC was adopted, the 5-year recurrence and survival rate still fell within the target as a whole.

Amongst five biomarkers, AFP seems to be the most reliable marker with the highest AUC value [Figure 2]. The usefulness of AFP in predicting recurrence after LT has been investigated by many researchers ${ }^{[12-14,30-32]}$, and AFP is incorporated in some selection ${ }^{[7,10,12,33]}$ and prognostic models ${ }^{[12-14]}$. The AFP model, developed by the Liver Transplantation French Study Group, combines serum AFP level, tumor size, and tumor number ${ }^{[12]}$. Another famous prognostic model is the RETREAT score, which incorporated microvascular invasion, tumor diameter, and tumor number other than the AFP value as prognostic variables ${ }^{[13]}$. Another prognostic model, the TRAIN score, incorporated the AFP slope, which was defined as [(final-AFP)(initial-AFP)]/time ${ }^{[14]}$. The cut-off value of AFP differs from study to study, ranging from $15 \mathrm{ng} / \mathrm{mL}$ to $1000 \mathrm{ng} / \mathrm{mL}^{[7,10,12,13,30-33]}$. The AFP cut-off value of $60 \mathrm{ng} / \mathrm{mL}$, used in the present study, is relatively low compared with those used in other studies, however, the cut-off value was shown to be useful in predicting recurrence [Supplementary Figure 2]. The present results as well as the previous reports justifiy the use of pretransplant AFP values in the expanded indication criteria of LT for HCC patients.

AFP-L3, a reliable marker for the diagnosis of $\mathrm{HCC}^{[20]}$, proved to be a promising marker for recurrence after LT since the specificity of AFP-L3 was the highest [Figure 2] and patient recurrence rate curves were well stratified using AFP-L3 [Supplementary Figure 2]. However, there has been little study ${ }^{[34]}$ investigating the usefulness of AFP-L3 in predicting HCC recurrence after LT. Highly sensitive AFP-L3 became available around 2010 in Japan, which enabled the measurement of AFP-L3 even in patients with total AFP levels below $20 \mathrm{ng} / \mathrm{mL}^{[20,35-37]}$. Highly sensitive AFP-L3 is reported to be 5-10 times more sensitive than conventional AFP-L3 ${ }^{[37]}$. Along with these studies in non-transplant HCC patients, the present results warrant further investigation and validation for the usefulness and efficacy of AFP-L3 in predicting HCC recurrence after LT.

The AUC value of DCP was the 3rd highest [Figure 2] and multivariate analysis revealed that DCP is one of the independent risk factors for recurrence [Table 2] in this cohort. Though DCP has not been commonly used in the west ${ }^{[38]}$, some argued that DCP is more predictive than $\mathrm{AFP}^{[8,39]}$, and indeed, DCP is incorporated in the extended indication criteria of LT at two major centers in Japan ${ }^{[6,8]}$. A new prognostic 
model was developed in Korea, i.e., the MoRAL score, using only serum levels of AFP and DCP ${ }^{[15]}$, which was shown to be more effective than the Milan criteria in predicting recurrence after LT. While DCP is criticized for not being a routine laboratory test in the West and for its dependence on vitamin $\mathrm{K}$ status and warfarin administration in clinical settings, reports from Asia as well as the present study warrant further study on the DCP in predicting HCC recurrence after LT.

NLR and PLR are indicators of inflammatory status previously reported as prognostic markers for the recurrence of various cancers, including $\mathrm{HCC}^{[16,17]}$. As the usefulness of NLR has been presented in both DDLT and LDLT settings ${ }^{[40,41]}$, NLR is incorporated in some prognostic models ${ }^{[14,42]}$. Although the usefulness of PLR has also been reported since 2012, supporting evidence is still limited ${ }^{[40,41]}$. NLR and PLR were not as useful as AFP, AFP-L3, and DCP in predicting HCC recurrence after LT in the present study [Figure 2, Table 2, Supplementary Figure 2]. One of the drawbacks of these inflammatory markers may be the inconstant nature of neutrophil, platelet, and lymphocyte counts. This is more so in cirrhotic patients who suffer from portal hypertension, splenomegaly, and consequently, pancytopenia. As for other biomarkers, we could not evaluate the usefulness of FDG-PET, one of the promising biomarkers reported previously ${ }^{[18,19]}$, because FDG-PET was not routinely performed at our institute.

Our analysis has several weaknesses related to its retrospective design and the limited number of patients included. Both the present and the national cohorts used in the establishment of the 5-5-500 criteria were based on the long time-course with a considerable number of cases from nearly 20 years ago. As the developments and advances in imaging modalities, anti-viral treatments, and immunosuppression regimens might have changed practice in the management of LT considerably over the last two decades, it seems mandatory to validate the criteria in the recent cohort or in the prospective study. Although the usefulness of tumor downstaging before LT has been reported recently ${ }^{[43]}$, unfortunately there was no case of intentional downstaging in the present cohort. In Japan, where the indication of LT for HCC is restricted to those with decompensated cirrhosis by the national insurance system, HCC patients with compensated cirrhosis are usually recommended for locoregional treatments and will be referred for LT when they develop decompensated cirrhosis not amenable to locoregional treatments. The downstaging strategy for those beyond the selection criteria and the expansion of the indication criteria are two opposite ways to expand the indication of LT for candidates, which should be compared and discussed in future studies.

In conclusion, the present study suggests that both the 5-5-500 criteria and the Japanese DEC are appropriate for patients with HCC in LDLT. AFP, including AFP-L3, was demonstrated to be a reliable biomarker and could reasonably be incorporated into the expanded selection criteria. Further validation with more recent cases and a prospective study is warranted.

\section{DECLARATIONS}

\section{Authors' contributions}

Conception and design: Ichida A, Akamatsu N

Provision of study materials or patients: Ichida A

Collection and assembly of data: Ichida A, Akamatsu N

Data analysis and interpretation: Ichida A, Akamatsu N

Manuscript writing: Ichida A, Akamatsu N, Hasegawa K

Final approval of manuscript: Ichida A, Akamatsu N, Hasegawa K

\section{Availability of data and materials}

The data used in the present study were submitted to the journal. 


\section{Financial support and sponsorship}

None.

\section{Conflicts of interest}

All authors declared that there are no conflicts of interest.

\section{Ethical approval and consent to participate}

The current study was approved as project number 2158-(6) by Graduate School of Medicine and Faculty of Medicine, the University of Tokyo Research Ethics Committee/Institutional Review Board. Informed consent was obtained in the form of opt-out on the web-site (http://www.u-tokyo-hbp-transplant-surgery. $\mathrm{jp} /)$.

\section{Consent for publication}

Not applicable.

\section{Copyright}

(C) The Author(s) 2020 .

\section{REFERENCES}

1. Mazzaferro V, Regalia E, Doci R, Andreola S, Pulvirenti A, et al. Liver transplantation for the treatment of small hepatocellular carcinomas in patients with cirrhosis. N Engl J Med 1996;334:693-9.

2. Yao FY, Ferrell L, Bass NM, Watson JJ, Bacchetti P, et al. Liver transplantation for hepatocellular carcinoma: expansion of the tumor size limits does not adversely impact survival. Hepatology 2001;33:1394-403.

3. Sugawara Y, Tamura S, Makuuchi M. Living donor liver transplantation for hepatocellular carcinoma: Tokyo University series. Dig Dis 2007;25:310-12.

4. Lee SG, Hwang S, Moon DB, Ahn CS, Kim KH, et al. Expanded indication criteria of living donor liver transplantation for hepatocellular carcinoma at one large-volume center. Liver Transpl 2008;14:935-45.

5. Mazzaferro V, Llovet JM, Miceli R, Bhoori S, Schiavo M, et al. Predicting survival after liver transplantation in patients with hepatocellular carcinoma beyond the Milan criteria: a retrospective, exploratory analysis. Lancet Oncol 2009;10:35-43.

6. Ito T, Takada Y, Ueda M, Haga H, Maetani Y, et al. Expansion of selection criteria for patients with hepatocellular carcinoma in living donor liver transplantation. Liver Transpl 2007;13:1637-44.

7. Toso C, Asthana S, Bigam DL, Shapiro AM, Kneteman NM. Reassessing selection criteria prior to liver transplantation for hepatocellular carcinoma utilizing the scientific registry of transplant recipients database. Hepatology 2008;49:832-8.

8. Taketomi A, Sanefuji K, Soejima Y, Yoshizumi T, Uchiyama H, et al. Impact of des-gamma-carboxy prothrombin and tumor size on the recurrence of hepatocellular carcinoma after living donor liver transplantation. Transplantation 2009;87:531-7.

9. DuBay D, Sandroussi C, Sandhu L, Cleary S, Guba M, et al. Liver transplantation for advanced hepatocellular carcinoma using poor tumor differentiation on biopsy as an exclusion criterion. Ann Surg 2011;253:166-72.

10. Kim JM, Kwon CH, Joh JW, Park JB, Lee JH, et al. Expanded criteria for liver transplantation in patients with hepatocellular carcinoma. Transplant Proc 2014;46:726-9.

11. Llovet JM. Expanding HCC criteria for liver transplant: the urgent need for prospective, robust data. Liver Transpl 2006;12:1741-3.

12. Duvoux C, Roudot Thoraval F, Decaens T, Pessione F, et al. Liver transplantation for hepatocellular carcinoma: a model including $\alpha$-fetoprotein improves the performance of Milan criteria. Gastroenterology 2012;143:986-94.

13. Mehta N, Heimbach J, Harnois DM, Sapisochin G, Dodge JL, et al. Validation of a risk estimation of tumor recurrence after transplant (RETREAT) score for hepatocellular carcinoma recurrence after liver transplant. JAMA Oncol 2017;3:493-500.

14. Lai Q, Nicolini D, Inostroza Nunez M, Iesari S, Goffette P, et al. A novel prognostic index in patients with hepatocellular cancer waiting for liver transplantation: Time-Radiological-response-Alpha-fetoprotein-INflammation (TRAIN) score. Ann Surg 2016;264:787-96.

15. Lee JH, Cho Y, Kim HY, Cho EJ, Lee DH, et al. Serum tumor markers provide refined prognostication in selecting liver transplantation candidate for hepatocellular carcinoma patients beyond the Milan criteria. Ann Surg 2016;263:842-50.

16. Halazun KJ, Hardy MA, Rana AA, Woodland DC 4th, Luyten EJ, et al. Negative impact of neutrophil-lymphocyte ratio on outcome after liver transplantation for hepatocellular carcinoma. Ann Surg 2009;250:141-51.

17. Zheng J, Cai J, Li H, Zeng K, He L, et al. Neutrophil to lymphocyte ratio and platelet to lymphocyte ratio as prognostic predictors for hepatocellular carcinoma patients with various treatments: a meta-analysis and systematic review. Cell Physiol Biochem 2017;44:967-81.

18. Hong G, Suh KS, Suh SW, Yoo T, Kim H, et al. Alpha-fetoprotein and (18)F-FDG positron emission tomography predict tumor recurrence better than Milan criteria in living donor liver transplantation. J Hepatol 2016;64:852-9.

19. Takada Y, Kaido T, Shirabe K, Nagano H, Egawa H, et al. Significance of preoperative fluorodeoxyglucose-positron emission tomography in prediction of tumor recurrence after liver transplantation for hepatocellular carcinoma patients: a Japanese multicenter study. J 
Hepatobiliary Pancreat Sci 2017;24:49-57.

20. Nakao K, Ichikawa T. Recent topics on alpha-fetoprotein. Hepatol Res 2013;43:820-5.

21. Cillo U, Giuliani T, Polacco M, Herrero Manley LM, Crivellari G, et al. Prediction of hepatocellular carcinoma biological behavior in patient selection for liver transplantation. World J Gastroenterol 2016;22:232-52.

22. Kobayashi T, Aikata H, Honda F, Nakano N, Nakamura Y, et al. Preoperative fluorine 18 fluorodeoxyglucose positron emission tomography/computed tomography for prediction of microvascular invasion in small hepatocellular carcinoma. J Comput Assist Tomogr 2016;40:524-30.

23. Imura S, Teraoku H, Yoshikawa M, Ishikawa D, Yamada S, et al. Potential predictive factors for microvascular invasion in hepatocellylar carcinoma classified within the Milan criteria. Int J Clin Oncol 2018;23:98-103.

24. Shimamura T, Akamatsu N, Fujiyoshi M, Kawaguchi A, Morita S, et al. Expanded living-donor liver transplantation criteria for patients with hepatocellular carcinoma based on the Japanese nationwide survey: the 5-5-500 rule - a retrospective study. Transpl Int 2019;32:356-68.

25. Kokudo N, Sugawara Y, Imamura H, Sano K, Makuuchi M. Tailoring the type of donor hepatectomy for adult living donor liver transplantation. Am J Transplant 2005;5:1694-703.

26. Akamatsu N, Sugawara Y, Kaneko J, Arita J, Sakamoto Y, et al. Low platelet counts and prolonged prothrombin time early after operation predict the 90 days morbidity and mortality in living-donor liver transplantation. Ann Surg 2017;265:166-72.

27. Furukawa H, Shimamura T, Suzuki T, Taniguchi M, Nakanishi K, et al. Liver transplantation for hepatocellular carcinoma: the Japanese experience. J Hepatobiliary Pancreat Sci 2010;17:533-8.

28. Tamura S, Sugawara Y, Kokudo N. Living donor liver transplantation for hepatocellular carcinoma: the Japanese experience. Oncology 2011;81:111-5.

29. Clavien PA, Lesurtel M, Bossuyt PM, Gores GJ, Langer B, et al. Recommendations for liver transplantation for hepatocellular carcinoma: an international consensus conference report. Lancet Oncol 2012;13:e11-22.

30. Todo S, Furukawa H, Tada M. Extending indication: role of living donor liver transplantation for hepatocellular carcinoma. Liver Transpl 2007;13:S48-54.

31. Mailey B, Artinyan A, Khalili J, Denitz J, Sanchez-Luege N, et al. Evaluation of absolute serum $\alpha$-fetoprotein levels in liver transplant for hepatocellular cancer. Arch Surg 2011;146:26-33.

32. Berry K, Ioannou GN. Serum alpha-fetoprotein level independently predicts posttransplant survival in patients with hepatocellular carcinoma. Liver Transpl 2013;19:634-45.

33. Zheng SS, Xu X, Wu J, Chen J, Wang WL, et al. Liver transplantation for hepatocellular carcinoma: Hangzhou experiences. Transplantation 2008;85:1726-32.

34. Wongjarupong N, Negron-Ocasio GM, Chaiteerakij R, Addissie BD, Mohamed EA, et al. Model combining pre-transplant tumor biomarkers and tumor size shows more utility in predicting hepatocellular carcinoma recurrence and survival than the BALAD models. World J Gastroenterol 2018;24:1321-31.

35. Tamura Y, Igarashi M, Kawai H, Suda T, Satomura S, et al. Clinical advantage of highly sensitive on-chip immunessay for fucosylated fraction of alpha-fetoprotein in patients with hepatocellular carcinoma. Dig Dis Sci 2010;55:3576-83.

36. Hanaoka T, Sato S, Tobita H, Miyake T, Ishihara S, et al. Clinical significance of the highly seisitive fucosylated fraction of alphafetoprotein in patients with chronic liver disease. J Gastroenterol Hepatol 2011;26:739-44.

37. Toyoda H, Kumada T, Tada T. Highly sensitive Lens clinaris agglutinin-reactive alpha-fetoprotein: a new tool for the management of hepatocellular carcinoma. Oncology 2011;81:61-5.

38. Olthoff KM, Goldberg DS. The MoRAL path beyond Milan. Ann Surg 2016;263:851-2.

39. Shirabe K, Itoh S, Yoshizumi T, Soejima Y, Taketomi A, et al. The predictors of microvascular invasion in candidates for liver transplantation with hepatocellular carcinoma-with special reference to the serum levels of des-gamma-carboxy prothrombin. J Surg Oncol 2007;95:235-40.

40. Rosenblatt RE, Tafesh ZH, Halazun KJ. Role of inflamematory markers as hepatocellular cancer selection tool in the setting of liver transplantation. Transl Gastroenterol Heaptol 2017;2:95.

41. Citores MJ, Lucena JL, de la Fuente S, Cuervas-Mons V. Serum biomarkers and risk of hepatocellular carcinoma recurrence after liver transplantation. World J Hepatol 2019;11:50-64.

42. Halazun KJ, Najjar M, Abdelmessih RM, Samstein B, Griesemer AD, et al. Recurrence after liver transplantation for hepatocellular carcinoma: a new MORAL to the story. Ann Surg 2017;265:557-64.

43. Mazzaferro V, Citterio D, Bhoori S, Bongini M, Miceli R, et al. Liver transplantation in hepatocellular carcinoma after tumour downstaging (XXL): a randomised, controlled, phase 2b/3 trial. Lancet Oncol 2020;21:947-56. 